wiesen sich nach Angaben der Centers for Disease Control and Prevention (CDC) 26 der in den USA zugelassenen Antibiotika als unwirksam. Die Frau starb letztlich an Multiorganversagen infolge einer Sepsis. Eine Chance hätte sie womöglich durch die Gabe des Antibiotikums Fosfomycin gehabt, so das CDC. Dieses ist in den USA jedoch nur zur oralen Behandlung einer unkomplizierten Zystitis zugelassen. Eine intravenöse Zubereitung, wie sie bei Knocheninfektionen nötig ist, gibt es nur in anderen Ländern - u. a. in Deutschland. Laut CDC ist eine solch ausgeprägte Resistenzlage gegen CRE dennoch ungewöhnlich.
Von über 250 untersuchten CRE-Fällen hätte bei etwa $80 \%$ wenigstens ein Aminoglykosid gewirkt und bei fast $90 \%$ das $\mathrm{Re}$ serveantibiotikum Tigecyclin. Das CDC nehmen jedoch diesen Fall zum Anlass, nochmals zu betonen, wie wichtig eine Isolierung von Patienten mit CRE-Infektion ist, um eine Verbreitung resistenter Keime zu unterbinden.

Erst im November hat das Europäische Präventionszentrum ECDC auf eine Zunahme von gegen Carbapenem resistenten Klebsiella pneumoniae hingewiesen. Der Anteil mit Resistenzen stieg europaweit von $4,6 \%$ in 2010 auf $8,3 \%$ in 2013 . Ruth Ney

\title{
Menopause beschleunigt Lufu-Verlust
}

\author{
Der Eintritt der Wechseljahre kann sich nachteilig auf die Lungenfunktion auswirken. \\ FVC und FEV 1 nehmen offenbar schneller ab als vor der Menopause.
}

Die Lungenfunktion wird mit dem Alter kontinuierlich schlechter. Dieser Prozess wird bekanntlich durch Rauchen stark beschleunigt. Neuen Erkenntnissen zufolge ist aber auch der Beginn der Menopause mit einer schnelleren Abnahme der Lungenfunktion assoziiert. Dies zeigt sich besonders deutlich bei der forcierten Vitalkapazität (FVC).

An der bevölkerungsbasierten Studie nahmen 1.438 Frauen teil, die zu Beginn zwischen 25 und 48 Jahre alt waren und knapp 20 Jahre beobachtet wurden (AJRCCM 2016; online 1. Dezember).

Die FVC reduzierte sich im Mittel jährlich um 15,9 ml und die Einsekundenkapazität $\left(\mathrm{FEV}_{1}\right)$ um $24,1 \mathrm{ml}$. Verglichen mit dem nicht-menopausalen Status war der FVC-Verlust in der Perimenopause um 10,2 ml und in der Postmenopause um 12,5 ml/ Jahr erhöht. Der zusätzliche $\mathrm{FEV}_{1}$-Verlust betrug 3,8 bzw. 5,2 ml/ Jahr. Laut den Studienautoren entsprechen die Einbußen bei der $\mathrm{FEV}_{1}$ der Wirkung von zwei Packungsjahren, die bei der FVC sogar dem Effekt von zehn Packungsjahren. Wurde berücksichtigt, dass die Frauen im Laufe der Jahre etwas schrumpften (um

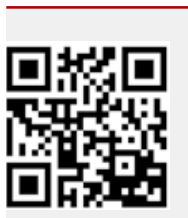
Apotheke in Schweden: Anti-RauchKampagne wird zum Internet-Hit

Ein Plakat, das Passanten „anhustet“? Die Idee einer schwedischen Apotheke klingt verrückt - schreckt Raucher mitten in Stockholm aber wirkungsvoll auf.

Der Mann auf der digitalen Leinwand, die die Apotheken-Kette „Apotek Hjärtat" für ihre Kampagne gegen das Rauchen entworfen hat, beginnt zu husten, sobald rauchende Passanten vorbeigehen: Die Installation an der Stockholmer U-Bahnstation Odenplan ist mit Rauchmeldern ausgestattet. Das Plakat sei an dieser Stelle platziert worden, da hier besonders viele Menschen rauchten, erklärt "Apotek Hjärtat“ in einem Facebook-Video. In dem Clip bleiben Raucher verdutzt stehen, als sie das Husten hören.

Die Apotheken-Kette hofft, dass die Schweden so ihre Neujahrsvorsätze leichter umsetzen können. Rund 376.000 Mal ist das Video auf Facebook bereits angesehen worden.
0,23 mm/Jahr), schwächte sich der Zusammenhang zwischen Menopause und Abnahme der Lungenfunktion ab. Der zusätzliche Verlust bei der FVC lag dann bei 7,7 $\mathrm{ml} / \mathrm{Jahr}$ in der Peri- und 9,0 $\mathrm{ml} / \mathrm{Jahr}$ in der Postmenopause. Die Unterschiede bei der $\mathrm{FEV}_{1}$ (-2,1 und $-2,6 \mathrm{ml} / \mathrm{Jahr})$ waren dann nicht mehr signifikant. „Der beschleunigte Verlust der Lungenfunktion zur Zeit der Menopause erklärt sich also teilweise durch eine Osteoporose", so die Studienautoren. Weil Osteoporose die Höhe der

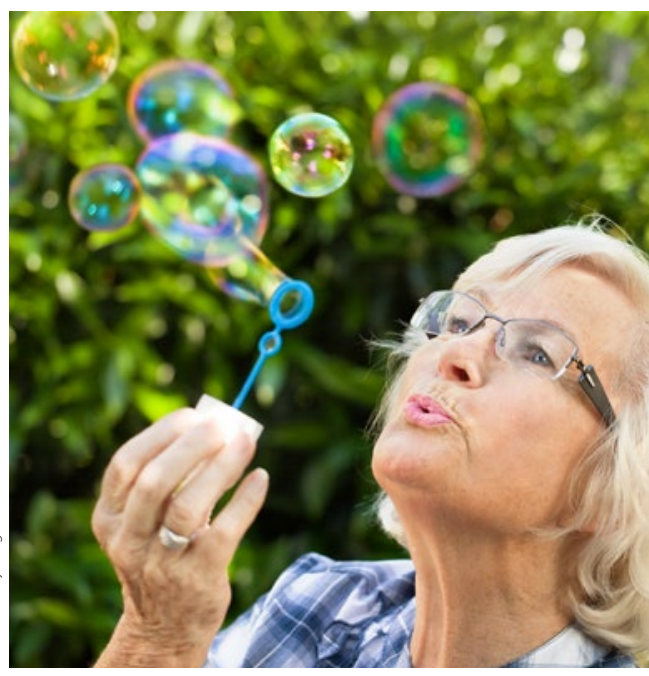

Entspanntes Pusten dürfte so mancher Frau nach der Menopause eher schwerer fallen. Dafür gibt's viele Gründe. Brustwirbel reduziert, könnte sie die Ausdehnung des Brustkorbs bei der Einatmung behindern und das Zwerchfell in eine suboptimale Position bringen.

Darüber hinaus vermuten sie einen direkten Zusammenhang zwischen hormonellen Veränderungen und Lungenfunktion. „Niedrige Estradiolspiegel sind mit einer vermehrten systemischen und pulmonalen Entzündung verbunden. "Entzündliche Prozesse wiederum würden mit niedrigeren FVC- und $\mathrm{FEV}_{1^{-}}$ Werten einhergehen.

Daher halten die Wissenschaflter es für wichtig, „dass Ärzten bewusst ist, dass sich die respiratorische Gesundheit mit der Menopause häufig verschlechtert“. Da die FVC stärker als die $\mathrm{FEV}_{1}$ von dem menopausenabhängigen Rückgang betroffen ist, liege die Ursache dafür offenbar weniger in einer Obstruktion als in einer verminderten Lungenentfaltung. Dr. Beate Schumacher 